\title{
Antibiotic Susceptibility Profiles of Bacteria from Diabetic Foot Infections in Selected Teaching Hospitals in Southwestern Nigeria
}

\author{
Olorunjuwon O. Bello ${ }^{1 *}$, Edward O. Oyekanmi ${ }^{1}$, Babatunde A. Kelly ${ }^{1}$, \\ Olakunle O. Mebude ${ }^{1}$ and Temitope K. Bello ${ }^{2}$ \\ ${ }^{1}$ College of Natural and Applied Sciences, Wesley University Ondo, Nigeria. \\ ${ }^{2}$ Faculty of Pure and Applied Sciences, Southwestern University Nigeria. \\ * Corresponding author email: juwonbello@yahoo.com
}

Received: 13 December 2017 / Revised: 12 January 2018 / Accepted: 18 January 2018 / Published: 28 January 2018

\begin{abstract}
One of the serious complications associated with diabetes is foot ulcer and this condition affects the quality of life in patients in all classes, races and ages. Chronic wounds are prone to colonization by wide array of microorganisms which could be extremely hazardous to patients if effective and timely therapeutic intervention is not made. This study was conducted to determine the antibiotic susceptibility profiles of bacteria from diabetic foot infections in southwestern Nigeria. Samples were collected from a total of 142 diabetic foot ulcer patients with moistened sterile cotton swabs. Nutrient agar, Mac-Conkey agar, blood agar and mannitol salt agar media were used for the isolation of total viable bacteria, Gram-negative non-spore forming lactose fermenters, fastidious bacteria and staphylococci, respectively. Morphological and biochemical characteristics of pure cultures were determined in accordance with standard laboratory criteria. API 20 E and API 20 NE were used for the confirmation of identity of the bacterial isolates. The disc diffusion technique was employed for the determination of antibiotic susceptibility of bacterial isolates in accordance with standard procedures. The antibiotics investigated included amikacin, amoxicillin, ampicillin, ceftazidime, cefazolin, ceftriaxone, chloramphenicol, ciprofloxacin, clindamycin, gentamicin, imipenem, linezolid, methicillin, netilmicin, ofloxacin, oxacillin, penicillin, piperacilin, sulfamethoxazole, trimethoprim and vancomycin. One hundred and seventyseven isolates were encountered and these were characterized into eleven bacterial species. These included Staphylococcus aureus (22.03\%), Psendomonas aeruginosa (16.95\%), Staphylococcus epidermidis (12.43\%), Proteus mirabilis (8.48\%), Klebsiella pneumoniae (7.91\%), E. coli (7.35\%), Staphylococcus saprophyticus (6.78\%), Streptococcus pyogenes (5.65\%), Morganella morganii (5.09\%), Citrobacterfreundii (4.52\%) and Acinetobacter baumannii (2.83\%). Gram-negative bacteria showed $76.99 \%$ susceptibility to the antibiotics while $22.85 \%$ was resistant. Gram-positive bacteria showed $93.75 \%$ susceptibility and $5.01 \%$ resistance to the antibiotics. This study revealed that there is no definite aetiologic bacterial agent for diabetic foot infections and many of the associated bacteria are sensitive to certain antibiotics.
\end{abstract}

Keywords: Antibiotics, bacteria, diabetes, foot ulcer, infections, resistance, susceptibility.

\section{Introduction}

Diabetes mellitus represents a major public health threat worldwide with an estimated prevalence in 2014 of 422 million patients [1]. A serious complication of diabetes is the development of foot ulcers. World Health Organization (WHO) considered diabetes as one of the twentieth-century epidemics and the most prevalent endocrine disease worldwide with about $10 \%$ of global adult population standing the risk of being affected [2]. This disease condition is associated with severe secondary and 
highly impairing pathologies which include vascular disease with high risk for heart failure and stroke, kidney failure requiring dialysis or transplantation, and blindness. The most devastating conditions in these patients include chronic wounds caused by an impaired immune response and an associated high microbial burden that frequently leads to amputation, mainly of the lower limbs [2], [3]. Every individual is prone to securing skin lesions during a lifetime and which usually heal up without any special care or treatment, but a simple tiny scratch could become a terrible condition for diabetic patients. The lesion could graduate into seriously infected chronic wound leading to amputation or even death [4], [5]. A contributing factor usually is skin microbiota of diabetic patients [6] - [10].

Naturally healing of diabetic chronic wounds is difficult and this makes some degree of therapeutic intervention imperative [11]. Corresponding clinical treatments are usually initiated after diagnosis of the wound chronic stage. Compromised tissues debridement, use of specialized dressings, and appropriate use of antibiotics are the most frequently used approaches to combating diabetic chronic wounds [12]. Success rates are, however, still not satisfactory as no significant improvement has been reported in more than fifty-percent of the patients to this approach. Hence, hospitalization and amputation were often the resulting outcome [2], [13], [14]. Inappropriate antibiotic administration could further impair wound healing progression in many patients [15], [16], and as physicians were left with no options, persistent administration systemic antibiotics becomes the order of the day [12], with the hope that something changes in the health condition of the patient that allows the wound to enter a remission stage [11]. There is no doubt that this is a lifethreatening problem which requires urgent chemotherapeutic interventions, hence the need to relentlessly determine the sensitivity of associated microorganisms to conventional antibiotics. Based on the severity and risk associated with foot ulcers, and variations of reports among different geographical places and periods, it becomes imperative to characterize and determine the antibiotic susceptibility patterns of the bacteria from diabetic foot infections in southwestern Nigeria.

Based on the severity and risk associated with foot ulcers, and variations of reports among different geographical places and periods, it becomes imperative to characterize and determine the antibiotic susceptibility patterns of the bacteria from diabetic foot infections in southwestern Nigeria.

\section{Materials and Methods}

\subsection{Collection of Samples}

Ethical clearances were obtained from the institutional ethical committees before collection of samples. Samples (pus, wound exudates) collections were undertaken in medical wards between October, 2016 and March, 2017. Wounds were washed vigorously with normal saline solution and discharges from margins and edges of ulcer were collected from a total of 142 patients (105 in-patients and 37 outpatients). Sixty-seven (67) samples were obtained from University College Hospital (UCH), Ibadan while 75 samples were obtained from Olabisi Onabanjo University Teaching Hospital (OOUTH), Sagamu. Samples were collected with sterile cotton swabs already moistened with sterile saline to prevent drying. The swabs were transported to the laboratory in sterile containers immediately after collection. Samples which were not analyzed within four hours of collection were discarded.

\subsection{Microbiological Analysis}

Swabs were separately inoculated on appropriate agar media for cultivation and enumeration of associated bacteria. Nutrient agar (Oxoid, England), MacConkey agar (Oxoid, England), blood agar (Oxoid, England) and mannitol salt agar (Oxoid, England) media were used for the isolation of total viable bacteria, Gram-negative non-spore forming lactose fermenters, fastidious bacteria and staphylococci, respectively. The inoculated plates were incubated at $37^{\circ} \mathrm{C}$ for 24 hours. Plates with mixed cultures were sub-cultured to obtain pure colonies of bacteria. Morphological and biochemical characteristics of the discreet colonies were determined in accordance with standard laboratory criteria. The tests performed include Gram staining, motility, catalase, oxidase, indole, methyl-red, VogesProskauer, urease, citrate utilization, starch hydrolysis, nitrate reduction and sugar fermentation test using glucose, sucrose, arabinose, maltose, xylose, galactose, sorbitol, invositol, raffinose and frauction while API $20 \mathrm{E}$ and API $20 \mathrm{NE}$ were used for the confirmation of identity of the bacterial isolates. 


\subsection{Antibiotic Susceptibility Test}

\subsubsection{Standardization of Inoculum}

Cultures of bacteria were cultivated on nutrient agar (Oxoid, England) plates and plates incubated for 24 hours at $37^{\circ} \mathrm{C}$. About $100 \mu \mathrm{l}$ of bacterial cells was dispensed in sterile normal saline to obtain the turbidity of $0.5 \mathrm{McF}$ arland standard, which is a solution of barium sulphate prepared from $0.6 \mathrm{ml}$ of $1 \%$ barium chloride added to $99.4 \mathrm{ml}$ of sulphuric acid [17], [18].

\subsubsection{Sensitivity Assay}

The disc diffusion technique was employed for the determination of antibiotic susceptibility of bacterial isolates in accordance with standard procedures. Young actively growing cultures of bacteria were obtained on nutrient agar (Oxoid, England) plates by overnight incubation at $37^{\circ} \mathrm{C}$. Muller Hinton agar (Oxoid, England) media was prepared in sterile Petri dishes. Sterilized swabs were dipped in overnight cultures and spread evenly over the media. The various antibiotic discs were aseptically placed over the media and incubated overnight at $37^{\circ} \mathrm{C}$. The conventional antibiotics investigated in this study included amikacin (30 $\mu \mathrm{g})$, amoxicillin (30 $\mu \mathrm{g})$, ampicillin $(10 \mu \mathrm{g})$, ceftazidime $(30 \mu \mathrm{g})$, cefazolin (30 $\mu \mathrm{g})$, ceftriaxone $(30 \mu \mathrm{g})$, chloramphenicol $(30 \mu \mathrm{g})$, ciprofloxacin $(5 \mu \mathrm{g})$,clindamycin $(2 \mu \mathrm{g})$, gentamicin (10 $\mu \mathrm{g})$, imipenem $(10 \mu \mathrm{g})$, linezolid (30 $\mu \mathrm{g})$, methicillin $(5 \mu \mathrm{g})$, netilmicin $(30 \mu \mathrm{g})$, ofloxacin (5 $\mu \mathrm{g})$, oxacillin $(2 \mu \mathrm{g})$, penicillin $(10 \mu \mathrm{g})$, piperacilin $(100 \mu \mathrm{g})$, sulfamethoxazole $(25 \mu \mathrm{g})$, trimethoprim (5 $\mu \mathrm{g})$ and vancomycin $(30 \mu \mathrm{g})$ [18].

\section{Results and Discussion}

A total of 142 samples, comprising of 105 in-patients and 37 out-patients, were collected. Out of the total samples, 75 (56 in-patients and 19 out-patients) were collected from UCH, Ibadan while 67 (49 in-patients and 18 out-patients) were obtained from OOUTH, Sagamu (Table 1). Table 2 showed the occurrence of bacteria among diabetic foot ulcer in- and outpatients visiting teaching hospitals in southwestern Nigeria. The organisms were distributed between the in-patients and out-patients visiting the teaching hospitals. Invariably, both the in-patients and outpatients contributed to the bacterial diversity encountered in this study. Table 3 showed the morphological and biochemical characteristics of bacteria isolated from diabetic foot ulcer in selected visiting hospitals in southwestern Nigeria. A total of one hundred and seventy-seven (177) isolates were encountered in this study and these were characterized as eleven (11) bacterial species. They were Acinetobacter baumannii, Citrobacter freundii, Escherichia coli, Klebsiella pneumoniae, Morganella morganii, Proteus mirabilis, Pseudomonas aeruginosa, Staphylococcus aureus, Staphylococcus epidermidis, Staphylococcus saprophyticus and Streptococcus pyogenes.

Table 1: Details of sample collection from patients from selected teaching hospitals in southwestern Nigeria

\begin{tabular}{|l|l|l|l|}
\hline Sampling Site & $\begin{array}{l}\text { In- } \\
\text { patient }\end{array}$ & $\begin{array}{l}\text { Out- } \\
\text { patient }\end{array}$ & $\begin{array}{l}\text { Total } \\
\text { Number of } \\
\text { Samples }\end{array}$ \\
\hline UCH, Ibadan & 56 & 19 & 75 \\
\hline $\begin{array}{l}\text { OOUTH, } \\
\text { Sagamu }\end{array}$ & 49 & 18 & 67 \\
\hline Total & 105 & 37 & 142 \\
\hline
\end{tabular}

Table 2: Occurrence of bacteria among diabetic foot ulcer in- and out-patients visiting teaching hospitals in southwestern Nigeria

\begin{tabular}{|c|c|c|c|}
\hline Bacteria & $\begin{array}{l}\text { In- } \\
\text { patient }\end{array}$ & $\begin{array}{l}\text { Out- } \\
\text { patient }\end{array}$ & $\begin{array}{l}\text { Total } \\
\text { number of } \\
\text { bacteria }\end{array}$ \\
\hline $\begin{array}{l}\text { Acinetobacter } \\
\text { baumannii }\end{array}$ & 4 & 1 & 5 \\
\hline Citrobacter freundii & 5 & 3 & 8 \\
\hline Escherichia coli & 9 & 4 & 13 \\
\hline Klebsiella pneumonia & 9 & 5 & 14 \\
\hline Morganella morganii & 7 & 2 & 9 \\
\hline Proteus mirabilis & 11 & 4 & 15 \\
\hline $\begin{array}{l}\text { Pseudomonas } \\
\text { aeruginosa }\end{array}$ & 23 & 7 & 30 \\
\hline Staphylococcus aureus & 30 & 9 & 39 \\
\hline $\begin{array}{l}\text { Staphylococcus } \\
\text { epidermidis }\end{array}$ & 17 & 5 & 22 \\
\hline $\begin{array}{l}\text { Staphylococcus } \\
\text { saprophyticus }\end{array}$ & 9 & 3 & 12 \\
\hline $\begin{array}{l}\text { Streptococcus } \\
\text { pyogenes }\end{array}$ & 6 & 4 & 10 \\
\hline Total & 130 & 47 & 177 \\
\hline
\end{tabular}


Table 3: Morphological and biochemical characteristics of bacteria isolated from diabetic foot ulcer patients visiting teaching hospitals in southwestern Nigeria

\begin{tabular}{|c|c|c|c|c|c|c|c|c|c|c|c|c|c|c|c|c|c|c|c|c|c|c|c|c|c|c|c|}
\hline 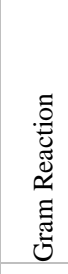 & 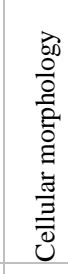 & $\begin{array}{l}\stackrel{\mathscr{J}}{\tilde{J}} \\
\underset{\tilde{J}}{\tilde{U}}\end{array}$ & 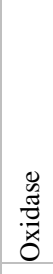 & $\frac{0}{0}$ & 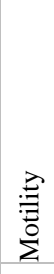 & $\frac{\overrightarrow{0}}{2}$ & 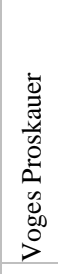 & 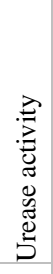 & 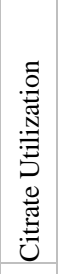 & 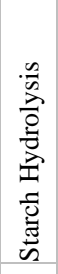 & 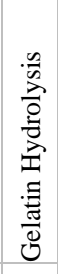 & 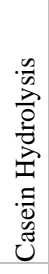 & 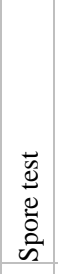 & 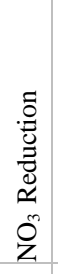 & 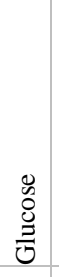 & 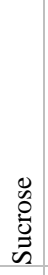 & 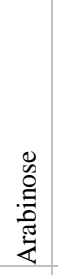 & 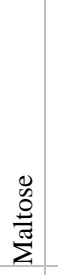 & 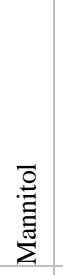 & $\frac{0}{\infty}$ & 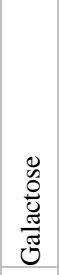 & 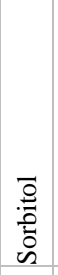 & $\begin{array}{l}\overline{\vec{O}} \\
\stackrel{\mathscr{n}}{0} \\
\stackrel{0}{\Xi}\end{array}$ & 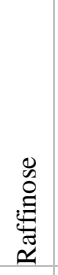 & 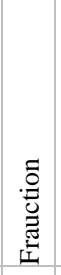 & 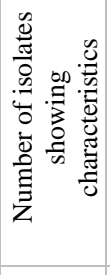 & $\begin{array}{l}\text { Most Probable } \\
\text { Identity }\end{array}$ \\
\hline -ve & $\mathrm{Cb}$ & + & - & - & - & - & - & + & + & - & - & - & - & - & + & - & - & - & - & + & - & - & - & - & - & 5 & $\begin{array}{l}\text { Acinetobacter } \\
\text { baumannii }\end{array}$ \\
\hline$+\mathrm{ve}$ & $\mathrm{R}$ & + & + & + & + & + & - & - & + & - & - & - & - & - & + & + & - & + & + & - & - & + & - & + & + & 8 & $\begin{array}{l}\text { Citrobacter } \\
\text { freundii }\end{array}$ \\
\hline -ve & $\mathrm{R}$ & - & + & - & + & + & + & - & + & - & - & + & + & + & + & + & + & - & + & + & - & - & - & - & + & 13 & Escherichia coli \\
\hline -ve & $\mathrm{R}$ & + & - & - & - & - & + & + & + & + & + & + & - & + & + & + & + & + & + & + & - & - & + & - & + & 14 & K. pneumoniae \\
\hline -ve & $\mathrm{R}$ & + & - & + & + & + & - & + & - & - & - & - & - & + & + & - & - & - & - & + & + & - & - & - & - & 9 & $\begin{array}{l}\text { Morganella } \\
\text { morganii }\end{array}$ \\
\hline -ve & $\mathrm{R}$ & + & + & - & + & - & + & + & + & - & + & - & - & + & + & + & + & + & + & + & + & - & - & + & + & 15 & P. aeruginosa \\
\hline -ve & $\mathrm{R}$ & + & - & - & + & + & - & + & + & + & - & - & - & + & + & - & - & - & - & + & - & - & - & - & - & 30 & Proteus mirabilis \\
\hline$+\mathrm{ve}$ & $\mathrm{C}$ & + & - & - & - & - & + & + & - & - & + & + & - & + & + & + & - & + & + & - & + & - & - & - & + & 39 & S. aureus \\
\hline$+\mathrm{ve}$ & $\mathrm{C}$ & + & - & - & - & - & + & + & - & - & + & - & - & - & + & + & - & - & - & - & - & - & - & - & + & 22 & S. epidermidis \\
\hline$+\mathrm{ve}$ & $\mathrm{C}$ & + & - & - & - & - & + & + & - & - & + & + & - & + & + & + & - & + & + & - & + & - & - & - & + & 12 & S. saprophyticus \\
\hline$+\mathrm{ve}$ & C & - & - & - & - & + & + & + & + & - & + & + & + & - & + & + & - & + & - & - & + & - & - & - & - & 10 & $\begin{array}{l}\text { Streptococcus } \\
\text { pyogenes }\end{array}$ \\
\hline
\end{tabular}

Keys: $\mathrm{Cb}=$ Coccobacilli $; \mathrm{R}=$ Rods $; \mathrm{C}=$ Cocci $;+=$ Positive reaction $;-=$ Negative reaction; $\mathrm{ND}=$ Not determined

The percentage occurrences of bacteria isolated from diabetic foot ulcer in-patients and out-patients were shown in Figure 1. Staphylococcus aureus had the highest percentage occurrence of $23.08 \%$ and $19.15 \%$ in inpatients and out-patients, respectively. This was followed by P. aeruginosa with $17.69 \%$ (in-patient) and $14.89 \%$ (out-patients). Among the in-patients, $S$. epidermidis had the next percentage occurrence of $13.08 \%$, followed by Proteus mirabilis (8.46\%), E. coli (6.92\%), S. saprophyticus (6.92\%), Morganella morganii $(5.39 \%)$, Streptococcus pyogenes $(4.62 \%)$, C. freundii $(3.85 \%)$ while Acinetobacter baumannii had the lowest occurrence of $3.08 \%$. However, among the outpatients, Acinetobacter baumannii with percentage occurrence of $12.13 \%$ was next to $P$. aeruginosa (14.89\%) while K. pneumoniae, S. epidermidis, E. coli, S. pyogenes, C. freundii and M. morganii had percentage occurrences of $10.64 \%, 10.64 \%, 8.51 \%, 8.51 \%$, $6.38 \%$ and $4.25 \%$, respectively.

The overall percentage occurrences of the bacterial species associated with diabetic foot ulcers was shown in Figure 2. S. aureus had the highest occurrence of $22.03 \%$, followed by $P$. aeruginosa $(16.95 \%), S$. epidermidis (12.43\%), P. mirabilis (8.48\%), K. pneumoniae (7.91\%), E. coli (7.35\%), S. saprophyticus (6.78\%), S. pyogenes (5.65\%), M. morganii $(5.09 \%)$, C. freundii (4.52\%) while $A$. baumannii $(2.83 \%)$ had the lowest occurrence. Percentage occurrence of Gram-positive and Gram-negative bacteria in diabetic foot ulcers was given in Figure 3. Results showed that there were a greater number of Gram-negative bacteria than Gram-positive bacteria associated with diabetic foot ulcers investigated in this study. The percentage occurrence of Gram-negative bacteria was 53\% while that of Gram-positive bacteria was $47 \%$. 


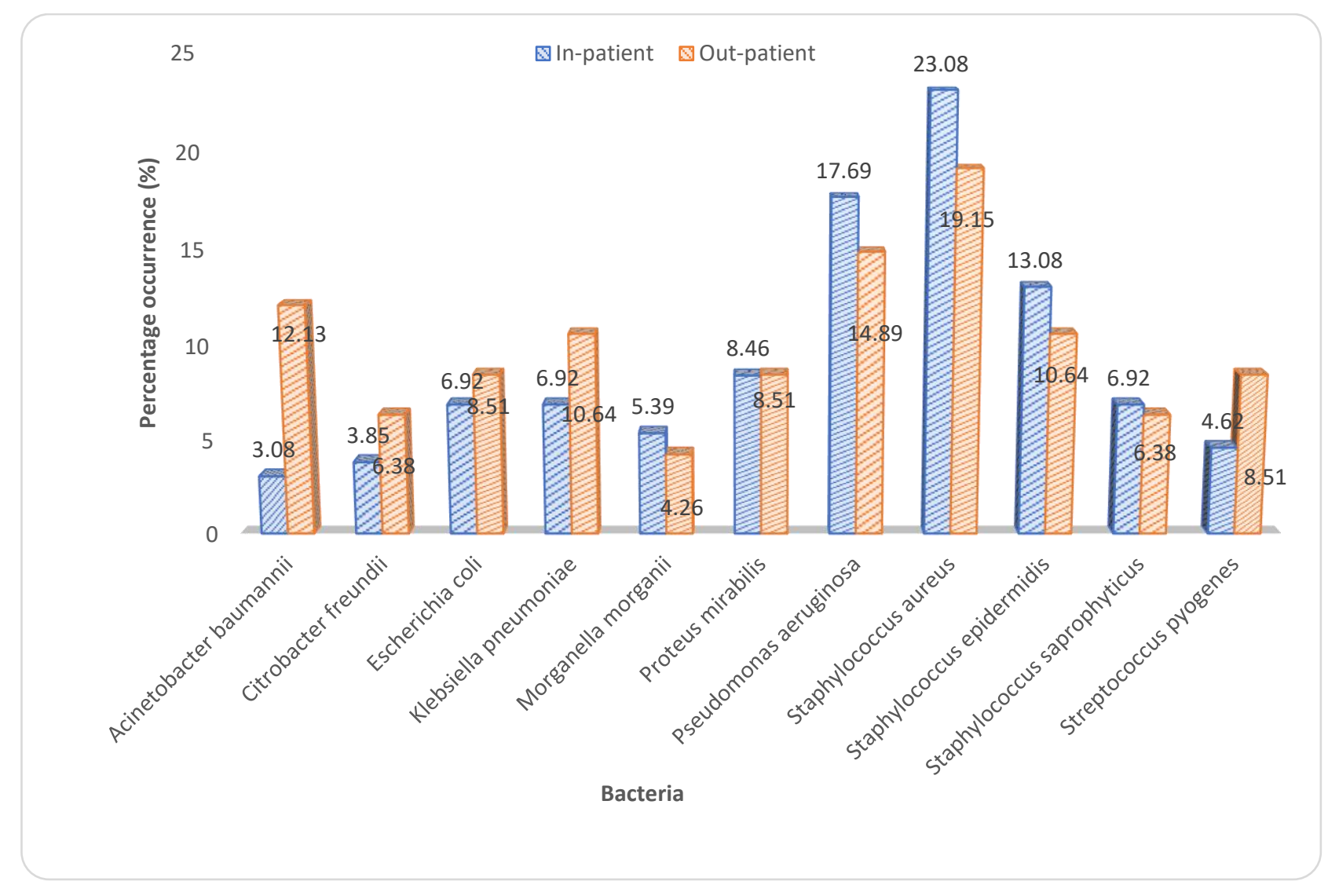

Figure 1: Percentage occurrence of bacteria isolated from diabetic foot ulcer in-patients and out-patients visiting teaching hospitals in southwestern Nigeria

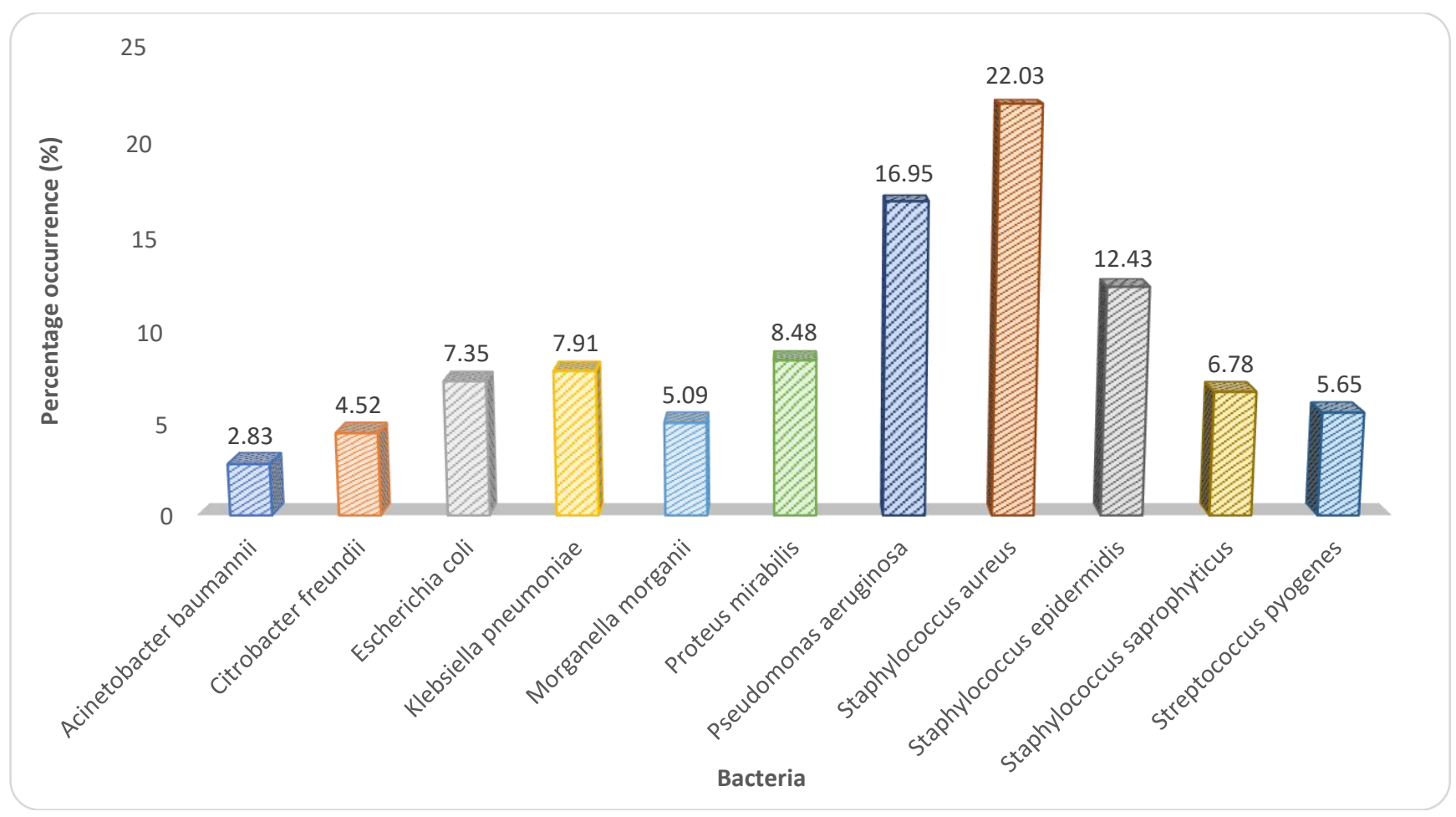

Figure 2: Overall percentage occurrence of bacteria associated with diabetic foot ulcer from patients visiting teaching hospitals in southwestern Nigeria 


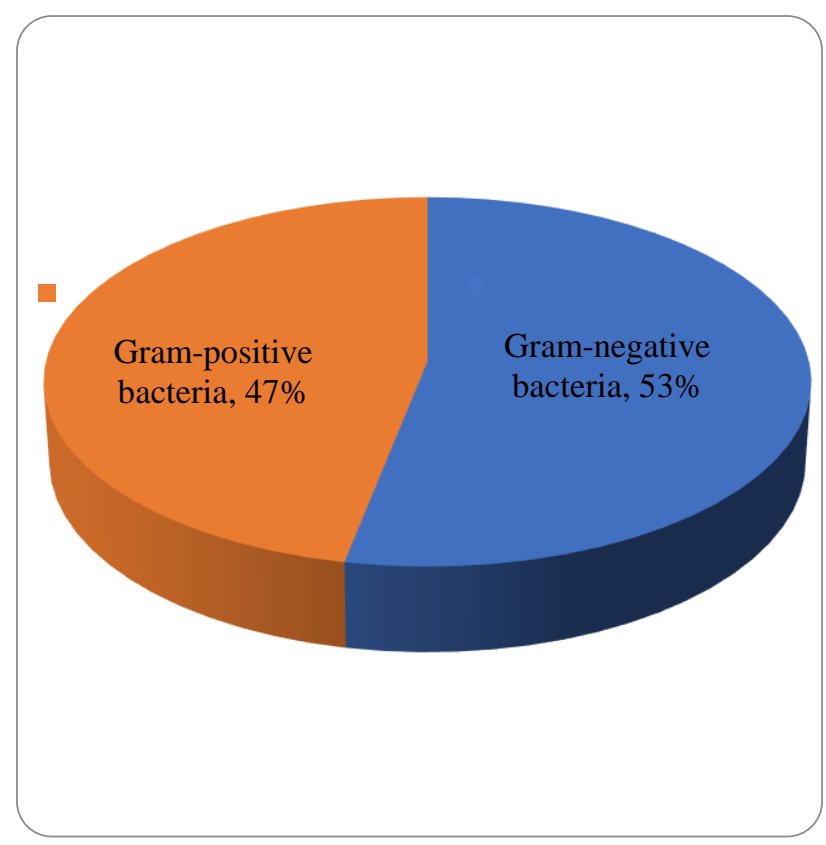

Figure 3: Percentage occurrence of Gram-positive and Gram-negative bacteria in diabetic foot ulcers from selected visiting hospitals in southwestern Nigeria

Tables $4 \mathrm{a}$ and $4 \mathrm{~b}$ showed the antibiotic susceptibility and resistance profiles of Gram-negative bacteria isolated from diabetic foot ulcers. Generally, most strains of Gram-negative bacterial species encountered in this study were susceptible to many of the antibiotics investigated. Acinetobacter baumannii showed 100\% susceptibility to amikacin, amoxicillin, ampicillin, ceftazidime, ceftriaxone, clindamycin, imipenem, methicillin and piperacilin 40\%, 20\%,60\% and $60 \%$ resistances were shown to chloramphenicol, gentamycin, netilmicin and ofloxacin, respectively. $C$. freundii showed $100 \%$ sensitivity to amikacin, ceftazidime, cefazolin, ceftriaxone, chloramphenicol, clindamycin, gentamycin, imipenem, methicillin, ofloxacin and piperacillin while $62.5 \%, 37.5 \%$ and $37.5 \%$ resistances were shown to amoxicillin, ampicillin and netilmicin, respectively. E. coli strains showed $100 \%, 84.6 \%, 84.6 \%, 15.4 \%$ and $15.4 \%$ resistances to amoxicillin, ampicillin, cefazolin, methicillin and netilmicin, respectively while 100\% susceptibility was shown to other antibiotics except amikacin to which $92.3 \%$ sensitivity and $7.7 \%$ intermediate sensitivity were shown. All strains of $K$. pneumoniae isolated were sensitive to amikacin, ceftazidime, ceftriaxone, clindamycin, gentamycin, imipenem, ofloxacin and piperacillin while all strains were resistant to amoxicillin and ampicillin. However, $64.3 \%, 21.4 \%, 28.6 \%$ and $28.6 \%$ of strains exhibited resistance to cefazolin, chloramphenicol, methicillin and netilmicin, respectively. All M. morganii strains were sensitive to all antibiotics except amoxicillin and ampicillin to which $22.2 \%$ and $44.4 \%$, resistances were shown respectively.

P. mirabilis exhibited $100 \%$ susceptibility to amikacin, ceftazidime, ceftriaxone, clindamycin, gentamycin, imipenem, methicillin, netilmicin, ofloxacin and piperacillin while strains showed $100 \%$ resistance to amoxicillin and ampicillin. However, the strains of organism exhibited $26.7 \%, 13.3 \%$ and $20 \%$ to cefazolin, chloramphenicol and nitilmicin, respectively. $P$. aeruginosa strains showed 100\% resistance to amoxicillin and ampicillin; $13.3 \%$, $63.3 \%, 60 \%, 23.3 \%, \quad 30 \%, 23.3 \%$ and $73.3 \%$ resistances were exhibited to amikacin, cefazolin, chloramphenicol, clindamycin, gentamycin, methicillin and netilmicin, respectively.

The antibiotic susceptibility and resistance profiles of Gram-positive bacteria isolated from diabetic foot ulcers were shown in Tables $5 \mathrm{a}$ and $5 \mathrm{~b}$. All $S$. aureus strains were sensitive to amikacin, amoxicillin, ampicillin, ceftriaxone, imipenem, linezolid, netilmicin, ofloxacin, penicillin, piperacillin, sulphamethazole and trimethoprim; strains showed $71.8 \%$ sensitivity and $28.2 \%$ intermediate sensitivity to vancomycin while $12.8 \%, 20.5 \%, 28.2 \%$ and $15.5 \%$ resistances were exhibited to ceftazidime, cefazolin, ciprofloxacin and gentamycin, respectively.

S. epidermidis strains showed $27.3 \%, 9.1 \%, 18.2 \%$ and $59.1 \%$ resistances to ampicillin, ceftazidime, penicillin and vancomycin, respectively while strains exhibited $100 \%$ sensitivity to other antibiotics. Strains of $S$. saprophyticus exhibited $25 \%, 16.7 \%$ and $66.7 \%$ resistances to penicillin, piperacillin and vancomycin, respectively; strains exhibited intermediate susceptibilities of $8.3 \%, 16.7 \%$ and $16.7 \%$ to ceftazidime, gentamycin and vancomycin, respectively while $100 \%$ susceptibility was shown to the remaining antibiotics. Strains of $S$. pyogenes were sensitive to all antibiotics except ampicillin, netilmicin and penicillin to which $30 \%, 10 \%$ and $30 \%$ resistances were shown, respectively while $20 \%$ of strains showed intermediate susceptibility to vancomycin. 
Table 4a: Antibiotic susceptibility and resistance profiles of Gram-negative bacteria isolated from diabetic foot ulcer patients visiting selected visiting hospitals in southwestern Nigeria

\begin{tabular}{|c|c|c|c|c|c|c|c|c|}
\hline Bacteria & & Amikacin & Amoxicillin & Ampicillin & Ceftazidime & Cefazolin & Ceftriaxone & $\begin{array}{l}\text { Chloram- } \\
\text { phenicol }\end{array}$ \\
\hline \multirow{3}{*}{$\begin{array}{l}\text { Acinetobacter } \\
\text { baumannii } \\
(\mathrm{n}=5)\end{array}$} & $\mathrm{S}$ & $\begin{array}{l}5 \\
100 \%\end{array}$ & $\begin{array}{l}5 \\
100 \%\end{array}$ & $\begin{array}{l}5 \\
100 \%\end{array}$ & $\begin{array}{l}5 \\
100 \%\end{array}$ & $\begin{array}{l}5 \\
100 \%\end{array}$ & $\begin{array}{l}5 \\
100 \%\end{array}$ & $\begin{array}{l}3 \\
60 \%\end{array}$ \\
\hline & I & 0 & 0 & 0 & 0 & 0 & 0 & 0 \\
\hline & $\mathrm{R}$ & 0 & 0 & 0 & 0 & 0 & 0 & $\begin{array}{l}2 \\
40 \%\end{array}$ \\
\hline \multirow[t]{3}{*}{$\begin{array}{l}\text { Citrobacter freundii } \\
(\mathrm{n}=8)\end{array}$} & $\mathrm{S}$ & $\begin{array}{l}8 \\
100 \%\end{array}$ & $\begin{array}{l}3 \\
37.5 \%\end{array}$ & $\begin{array}{l}5 \\
62.5 \%\end{array}$ & $\begin{array}{l}8 \\
100 \%\end{array}$ & $\begin{array}{l}8 \\
100 \%\end{array}$ & $\begin{array}{l}8 \\
100 \%\end{array}$ & $\begin{array}{l}8 \\
100 \%\end{array}$ \\
\hline & I & 0 & 0 & 0 & 0 & 0 & 0 & 0 \\
\hline & $\mathrm{R}$ & 0 & $\begin{array}{l}5 \\
62.5 \%\end{array}$ & $\begin{array}{l}3 \\
37.5 \%\end{array}$ & 0 & 0 & 0 & 0 \\
\hline \multirow[t]{3}{*}{$\begin{array}{l}\text { Escherichia coli } \\
(\mathrm{n}=13)\end{array}$} & $\mathrm{S}$ & $\begin{array}{l}12 \\
92.3 \%\end{array}$ & 0 & $\begin{array}{l}2 \\
15.4 \%\end{array}$ & $\begin{array}{l}13 \\
100 \%\end{array}$ & $\begin{array}{l}2 \\
15.4 \%\end{array}$ & $\begin{array}{l}13 \\
100 \%\end{array}$ & $\begin{array}{l}13 \\
100 \%\end{array}$ \\
\hline & I & $\begin{array}{l}1 \\
7.7 \%\end{array}$ & 0 & 0 & 0 & 0 & 0 & 0 \\
\hline & $\mathrm{R}$ & 0 & $\begin{array}{l}13 \\
100 \%\end{array}$ & $\begin{array}{l}11 \\
84.6 \%\end{array}$ & 0 & $\begin{array}{l}11 \\
84.6 \%\end{array}$ & 0 & 0 \\
\hline \multirow{3}{*}{$\begin{array}{l}\text { Klebsiella } \\
\text { pneumoniae } \\
(\mathrm{n}=14)\end{array}$} & $\mathrm{S}$ & $\begin{array}{l}14 \\
100 \%\end{array}$ & 0 & 0 & $\begin{array}{l}14 \\
100 \%\end{array}$ & $\begin{array}{l}5 \\
35.7 \%\end{array}$ & $\begin{array}{l}14 \\
100 \%\end{array}$ & $\begin{array}{l}11 \\
78.6 \%\end{array}$ \\
\hline & I & 0 & 0 & 0 & 0 & 0 & 0 & 0 \\
\hline & $\mathrm{R}$ & 0 & $\begin{array}{l}14 \\
100 \%\end{array}$ & $\begin{array}{l}14 \\
100 \%\end{array}$ & 0 & $\begin{array}{l}9 \\
64.3 \%\end{array}$ & 0 & $\begin{array}{l}3 \\
21.4 \%\end{array}$ \\
\hline \multirow{3}{*}{$\begin{array}{l}\text { Morganella } \\
\text { morganii } \\
(\mathrm{n}=9)\end{array}$} & $\mathrm{S}$ & $\begin{array}{l}9 \\
100 \%\end{array}$ & $\begin{array}{l}7 \\
77.8\end{array}$ & $\begin{array}{l}5 \\
55.6 \%\end{array}$ & $\begin{array}{l}9 \\
100 \%\end{array}$ & $\begin{array}{l}9 \\
100 \%\end{array}$ & $\begin{array}{l}9 \\
100 \%\end{array}$ & $\begin{array}{l}9 \\
100 \%\end{array}$ \\
\hline & I & 0 & 0 & 0 & 0 & 0 & 0 & 0 \\
\hline & $\mathrm{R}$ & 0 & $\begin{array}{l}2 \\
22.2 \%\end{array}$ & $\begin{array}{l}4 \\
44.4 \%\end{array}$ & 0 & 0 & 0 & 0 \\
\hline \multirow[t]{3}{*}{$\begin{array}{l}\text { Proteus mirabilis } \\
(\mathrm{n}=15)\end{array}$} & $\mathrm{S}$ & $\begin{array}{l}15 \\
100 \%\end{array}$ & 0 & 0 & $\begin{array}{l}15 \\
100 \%\end{array}$ & $\begin{array}{l}11 \\
73.3 \%\end{array}$ & $\begin{array}{l}15 \\
100 \%\end{array}$ & $\begin{array}{l}13 \\
86.7 \%\end{array}$ \\
\hline & I & 0 & 0 & 0 & 0 & 0 & 0 & 0 \\
\hline & $\mathrm{R}$ & 0 & $\begin{array}{l}15 \\
100 \%\end{array}$ & $\begin{array}{l}15 \\
100 \%\end{array}$ & 0 & $\begin{array}{l}4 \\
26.7 \%\end{array}$ & 0 & $\begin{array}{l}2 \\
13.3 \%\end{array}$ \\
\hline \multirow{3}{*}{$\begin{array}{l}\text { Pseudomonas } \\
\text { aeruginosa } \\
(\mathrm{n}=30)\end{array}$} & $\mathrm{S}$ & $\begin{array}{l}25 \\
83.3 \%\end{array}$ & 0 & 0 & $\begin{array}{l}30 \\
100 \%\end{array}$ & $\begin{array}{l}11 \\
36.7 \%\end{array}$ & $\begin{array}{l}30 \\
100 \%\end{array}$ & $\begin{array}{l}12 \\
40 \%\end{array}$ \\
\hline & I & $\begin{array}{l}1 \\
3.3 \%\end{array}$ & 0 & 0 & 0 & 0 & 0 & 0 \\
\hline & $\mathrm{R}$ & $\begin{array}{l}4 \\
13.3 \%\end{array}$ & $\begin{array}{l}30 \\
100 \%\end{array}$ & $\begin{array}{l}30 \\
100 \%\end{array}$ & 0 & $\begin{array}{l}19 \\
63.3 \%\end{array}$ & 0 & $\begin{array}{l}18 \\
60 \%\end{array}$ \\
\hline
\end{tabular}

Keys: $\mathrm{S}=$ Susceptible or Sensitive; I = Intermediately Susceptible/Sensitive and R = Resistant 
Antibiotic Susceptibility Profiles of Bacteria from Diabetic Foot Infections in Selected Teaching Hospitals in Southwestern Nigeria

Table 4b: Antibiotic susceptibility and resistance profiles of Gram-negative bacteria isolated from diabetic foot ulcer patients visiting selected visiting hospitals in southwestern Nigeria

\begin{tabular}{|c|c|c|c|c|c|c|c|c|}
\hline Bacteria & & Clindamycin & Gentamycin & Imipenem & Methicillin & Netilmicin & Ofloxacin & Piperacilin \\
\hline \multirow{3}{*}{$\begin{array}{l}\text { Acinetobacter } \\
\text { baumannii } \\
(\mathrm{n}=5)\end{array}$} & $\mathrm{S}$ & $\begin{array}{l}5 \\
100 \%\end{array}$ & $\begin{array}{l}4 \\
80 \%\end{array}$ & $\begin{array}{l}5 \\
100 \%\end{array}$ & $\begin{array}{l}5 \\
100 \%\end{array}$ & $\begin{array}{l}2 \\
40 \%\end{array}$ & $\begin{array}{l}2 \\
40 \%\end{array}$ & $\begin{array}{l}5 \\
100 \%\end{array}$ \\
\hline & I & 0 & 0 & 0 & 0 & 0 & 0 & 0 \\
\hline & $\mathrm{R}$ & 0 & $\begin{array}{l}1 \\
20 \%\end{array}$ & 0 & 0 & $\begin{array}{l}3 \\
60 \%\end{array}$ & $\begin{array}{l}3 \\
60 \%\end{array}$ & 0 \\
\hline \multirow[t]{3}{*}{$\begin{array}{l}\text { Citrobacter freundii } \\
(\mathrm{n}=8)\end{array}$} & $\mathrm{S}$ & $\begin{array}{l}8 \\
100 \%\end{array}$ & $\begin{array}{l}8 \\
100 \%\end{array}$ & $\begin{array}{l}8 \\
100 \%\end{array}$ & $\begin{array}{l}8 \\
100 \%\end{array}$ & $\begin{array}{l}5 \\
62.5 \%\end{array}$ & $\begin{array}{l}8 \\
100 \%\end{array}$ & $\begin{array}{l}8 \\
100 \%\end{array}$ \\
\hline & I & 0 & 0 & 0 & 0 & 0 & 0 & 0 \\
\hline & $\mathrm{R}$ & 0 & 0 & 0 & 0 & $\begin{array}{l}3 \\
37.5 \%\end{array}$ & 0 & 0 \\
\hline \multirow[t]{3}{*}{$\begin{array}{l}\text { Escherichia coli } \\
(\mathrm{n}=13)\end{array}$} & $\mathrm{S}$ & $\begin{array}{l}13 \\
100 \%\end{array}$ & $\begin{array}{l}13 \\
100 \%\end{array}$ & $\begin{array}{l}13 \\
100 \%\end{array}$ & $\begin{array}{l}11 \\
84.6 \%\end{array}$ & $\begin{array}{l}11 \\
84.6 \%\end{array}$ & $\begin{array}{l}13 \\
100 \%\end{array}$ & $\begin{array}{l}13 \\
100 \%\end{array}$ \\
\hline & I & 0 & 0 & 0 & 0 & 0 & 0 & 0 \\
\hline & $\mathrm{R}$ & 0 & 0 & 0 & $\begin{array}{l}2 \\
15.4 \%\end{array}$ & $\begin{array}{l}2 \\
15.4 \%\end{array}$ & 0 & 0 \\
\hline \multirow{2}{*}{$\begin{array}{l}\text { Klebsiella } \\
\text { pneumoniae } \\
(\mathrm{n}=14)\end{array}$} & I & 0 & 0 & 0 & 0 & 0 & 0 & 0 \\
\hline & $\mathrm{R}$ & 0 & 0 & 0 & $\begin{array}{l}4 \\
28.6 \%\end{array}$ & $\begin{array}{l}4 \\
28.6 \%\end{array}$ & 0 & 0 \\
\hline \multirow{3}{*}{$\begin{array}{l}\text { Morganella } \\
\text { morganii } \\
(\mathrm{n}=9)\end{array}$} & $\mathrm{S}$ & $\begin{array}{l}9 \\
100 \%\end{array}$ & $\begin{array}{l}9 \\
100 \%\end{array}$ & $\begin{array}{l}9 \\
100 \%\end{array}$ & $\begin{array}{l}9 \\
100 \%\end{array}$ & $\begin{array}{l}9 \\
100 \%\end{array}$ & $\begin{array}{l}9 \\
100 \%\end{array}$ & $\begin{array}{l}9 \\
100 \%\end{array}$ \\
\hline & I & 0 & 0 & 0 & 0 & 0 & 0 & 0 \\
\hline & $\mathrm{R}$ & 0 & 0 & 0 & 0 & 0 & 0 & 0 \\
\hline \multirow[t]{3}{*}{$\begin{array}{l}\text { Proteus mirabilis } \\
(\mathrm{n}=15)\end{array}$} & $\mathrm{S}$ & $\begin{array}{l}15 \\
100 \%\end{array}$ & $\begin{array}{l}15 \\
100 \%\end{array}$ & $\begin{array}{l}15 \\
100 \%\end{array}$ & $\begin{array}{l}15 \\
100 \%\end{array}$ & $\begin{array}{l}12 \\
80 \%\end{array}$ & $\begin{array}{l}15 \\
100 \%\end{array}$ & $\begin{array}{l}15 \\
100 \%\end{array}$ \\
\hline & I & 0 & 0 & 0 & 0 & 0 & 0 & 0 \\
\hline & $\mathrm{R}$ & 0 & 0 & 0 & 0 & $\begin{array}{l}3 \\
20 \%\end{array}$ & 0 & 0 \\
\hline \multirow{2}{*}{$\begin{array}{l}\text { Pseudomonas } \\
\text { aeruginosa } \\
(\mathrm{n}=30)\end{array}$} & $\mathrm{S}$ & $\begin{array}{l}23 \\
76.7 \%\end{array}$ & $\begin{array}{l}21 \\
70 \%\end{array}$ & $\begin{array}{l}30 \\
100 \%\end{array}$ & $\begin{array}{l}23 \\
76.7 \%\end{array}$ & $\begin{array}{l}8 \\
26.7 \%\end{array}$ & $\begin{array}{l}30 \\
100 \%\end{array}$ & $\begin{array}{l}30 \\
100 \%\end{array}$ \\
\hline & I & 0 & 0 & 0 & 0 & 0 & 0 & 0 \\
\hline
\end{tabular}

Keys: $\mathrm{S}=$ Susceptible or Sensitive; $\mathrm{I}=$ Intermediately Susceptible/Sensitive and $\mathrm{R}=$ Resistant

Table 5a: Antibiotic susceptibility and resistance profiles of Gram-positive bacteria isolated from diabetic foot ulcer patients visiting selected visiting hospitals in southwestern Nigeria

\begin{tabular}{|c|c|c|c|c|c|c|c|c|c|c|}
\hline Bacteria & & $\begin{array}{l}\text { Ami- } \\
\text { kacin }\end{array}$ & $\begin{array}{l}\text { Amoxi- } \\
\text { cillin }\end{array}$ & $\begin{array}{l}\text { Ampi- } \\
\text { cillin }\end{array}$ & $\begin{array}{l}\text { Cefta- } \\
\text { zidime }\end{array}$ & Cefa-zolin & $\begin{array}{l}\text { Ceftria- } \\
\text { xone }\end{array}$ & $\begin{array}{l}\text { Cipro- } \\
\text { floxacin }\end{array}$ & $\begin{array}{l}\text { Genta- } \\
\text { mycin }\end{array}$ & Imipenam \\
\hline \multirow[t]{3}{*}{$\begin{array}{l}\text { S. aureus } \\
(\mathrm{n}=39)\end{array}$} & $\mathrm{S}$ & $\begin{array}{l}39 \\
100 \%\end{array}$ & $\begin{array}{l}39 \\
100 \%\end{array}$ & $\begin{array}{l}39 \\
100 \%\end{array}$ & $\begin{array}{l}34 \\
87.2 \%\end{array}$ & $\begin{array}{l}31 \\
79.5 \%\end{array}$ & $\begin{array}{l}39 \\
100 \%\end{array}$ & $\begin{array}{l}28 \\
71.8 \%\end{array}$ & $\begin{array}{l}33 \\
84.6 \%\end{array}$ & $\begin{array}{l}39 \\
100 \%\end{array}$ \\
\hline & I & 0 & 0 & 0 & 0 & 0 & 0 & 0 & 0 & 0 \\
\hline & $\mathrm{R}$ & 0 & 0 & 0 & $\begin{array}{l}5 \\
12.8 \%\end{array}$ & $\begin{array}{l}8 \\
20.5 \%\end{array}$ & 0 & $\begin{array}{l}11 \\
28.2 \%\end{array}$ & $\begin{array}{l}6 \\
15.5 \%\end{array}$ & 0 \\
\hline \multirow[t]{3}{*}{$\begin{array}{l}\text { S. epidermidis } \\
(\mathrm{n}=22)\end{array}$} & $\mathrm{S}$ & $\begin{array}{l}22 \\
100 \%\end{array}$ & $\begin{array}{l}22 \\
100 \%\end{array}$ & $\begin{array}{l}16 \\
72.7 \%\end{array}$ & $\begin{array}{l}20 \\
90.9 \%\end{array}$ & $\begin{array}{l}22 \\
100 \%\end{array}$ & $\begin{array}{l}22 \\
100 \%\end{array}$ & $\begin{array}{l}22 \\
100 \%\end{array}$ & $\begin{array}{l}22 \\
100 \%\end{array}$ & $\begin{array}{l}22 \\
100 \%\end{array}$ \\
\hline & I & 0 & 0 & 0 & 0 & 0 & 0 & 0 & 0 & 0 \\
\hline & $\mathrm{R}$ & 0 & 0 & $\begin{array}{l}6 \\
27.3 \%\end{array}$ & $\begin{array}{l}2 \\
9.1 \%\end{array}$ & 0 & 0 & 0 & 0 & 0 \\
\hline \multirow[t]{3}{*}{$\begin{array}{l}\text { S. saprophyticus } \\
(\mathrm{n}=12)\end{array}$} & $\mathrm{S}$ & $\begin{array}{l}12 \\
100 \%\end{array}$ & $\begin{array}{l}12 \\
100 \%\end{array}$ & $\begin{array}{l}12 \\
100 \%\end{array}$ & $\begin{array}{l}11 \\
91.7 \%\end{array}$ & $\begin{array}{l}12 \\
100 \%\end{array}$ & $\begin{array}{l}12 \\
100 \%\end{array}$ & $\begin{array}{l}12 \\
100 \%\end{array}$ & $\begin{array}{l}10 \\
83.3 \%\end{array}$ & $\begin{array}{l}12 \\
100 \%\end{array}$ \\
\hline & I & 0 & 0 & 0 & $\begin{array}{l}1 \\
8.3 \%\end{array}$ & 0 & 0 & 0 & $\begin{array}{l}2 \\
16.7 \%\end{array}$ & 0 \\
\hline & $\mathrm{R}$ & 0 & 0 & 0 & 0 & 0 & 0 & 0 & 0 & 0 \\
\hline \multirow[t]{3}{*}{$\begin{array}{l}\text { S. pyogenes } \\
(\mathrm{n}=10)\end{array}$} & $\mathrm{S}$ & $\begin{array}{l}10 \\
100 \%\end{array}$ & $\begin{array}{l}10 \\
100 \%\end{array}$ & $\begin{array}{l}7 \\
70 \%\end{array}$ & $\begin{array}{l}10 \\
100 \%\end{array}$ & $\begin{array}{l}10 \\
100 \%\end{array}$ & $\begin{array}{l}10 \\
100 \%\end{array}$ & $\begin{array}{l}10 \\
100 \%\end{array}$ & $\begin{array}{l}10 \\
100 \%\end{array}$ & $\begin{array}{l}10 \\
100 \%\end{array}$ \\
\hline & I & 0 & 0 & 0 & 0 & 0 & 0 & 0 & 0 & 0 \\
\hline & $\mathrm{R}$ & 0 & 0 & $\begin{array}{l}3 \\
30 \%\end{array}$ & 0 & 0 & 0 & 0 & 0 & 0 \\
\hline
\end{tabular}

Keys: S = Susceptible or Sensitive; I = Intermediately Susceptible/Sensitive and R = Resistant 
Bello et al., Int. Ann. Sci.; Vol. 4 Issue 1, pp: 1-13, 2018

Table 5b: Antibiotic susceptibility and resistance profiles of Gram-positive bacteria isolated from diabetic foot ulcer patients visiting selected visiting hospitals in southwestern Nigeria

\begin{tabular}{|c|c|c|c|c|c|c|c|c|c|c|}
\hline Bacteria & & $\begin{array}{l}\text { Line- } \\
\text { zolid }\end{array}$ & $\begin{array}{l}\text { Netil- } \\
\text { micin }\end{array}$ & $\begin{array}{l}\text { Oflo- } \\
\text { xacin }\end{array}$ & $\begin{array}{l}\text { Oxa- } \\
\text { cillin }\end{array}$ & $\begin{array}{l}\text { Peni- } \\
\text { cillin }\end{array}$ & Pipera-cilin & $\begin{array}{l}\text { Sulpha- } \\
\text { methazole }\end{array}$ & $\begin{array}{l}\text { Trime- } \\
\text { thoprim }\end{array}$ & $\begin{array}{l}\text { Vanco- } \\
\text { mycin }\end{array}$ \\
\hline \multirow[t]{3}{*}{$\begin{array}{l}\text { S. aureus } \\
(\mathrm{n}=39)\end{array}$} & $\mathrm{S}$ & $\begin{array}{l}39 \\
100 \%\end{array}$ & $\begin{array}{l}39 \\
100 \%\end{array}$ & $\begin{array}{l}39 \\
100 \%\end{array}$ & $\begin{array}{l}34 \\
87.2 \%\end{array}$ & $\begin{array}{l}39 \\
100 \%\end{array}$ & $\begin{array}{l}39 \\
100 \%\end{array}$ & $\begin{array}{l}39 \\
100 \%\end{array}$ & $\begin{array}{l}39 \\
100 \%\end{array}$ & $\begin{array}{l}28 \\
71.8 \%\end{array}$ \\
\hline & I & 0 & 0 & 0 & 0 & 0 & 0 & 0 & 0 & $\begin{array}{l}11 \\
28.2 \%\end{array}$ \\
\hline & $\mathrm{R}$ & 0 & 0 & 0 & 5 & 0 & 0 & 0 & 0 & 0 \\
\hline \multirow[t]{3}{*}{$\begin{array}{l}\text { S. epidermidis } \\
(\mathrm{n}=22)\end{array}$} & $\mathrm{S}$ & $\begin{array}{l}22 \\
100 \%\end{array}$ & $\begin{array}{l}22 \\
100 \%\end{array}$ & $\begin{array}{l}22 \\
100 \%\end{array}$ & $\begin{array}{l}22 \\
100 \%\end{array}$ & $\begin{array}{l}18 \\
81.8 \%\end{array}$ & $\begin{array}{l}22 \\
100 \%\end{array}$ & $\begin{array}{l}22 \\
100 \%\end{array}$ & $\begin{array}{l}22 \\
100 \%\end{array}$ & $\begin{array}{l}9 \\
40.9 \%\end{array}$ \\
\hline & I & 0 & 0 & 0 & 0 & 0 & 0 & 0 & 0 & 0 \\
\hline & $\mathrm{R}$ & 0 & 0 & 0 & 0 & $\begin{array}{l}4 \\
18.2 \%\end{array}$ & 0 & 0 & 0 & $\begin{array}{l}13 \\
59.1 \%\end{array}$ \\
\hline \multirow[t]{3}{*}{$\begin{array}{l}\text { S. saprophyticus } \\
(\mathrm{n}=12)\end{array}$} & $\mathrm{S}$ & $\begin{array}{l}12 \\
100 \%\end{array}$ & $\begin{array}{l}12 \\
100 \%\end{array}$ & $\begin{array}{l}12 \\
100 \%\end{array}$ & $\begin{array}{l}12 \\
100 \%\end{array}$ & $\begin{array}{l}9 \\
66.7 \%\end{array}$ & $\begin{array}{l}10 \\
83.3 \%\end{array}$ & $\begin{array}{l}12 \\
100 \%\end{array}$ & $\begin{array}{l}12 \\
100 \%\end{array}$ & $\begin{array}{l}2 \\
16.7 \%\end{array}$ \\
\hline & I & 0 & 0 & 0 & 0 & 0 & 0 & 0 & 0 & $\begin{array}{l}2 \\
16.7 \%\end{array}$ \\
\hline & $\mathrm{R}$ & 0 & 0 & 0 & 0 & $\begin{array}{l}3 \\
25 \%\end{array}$ & $\begin{array}{l}2 \\
16.7 \%\end{array}$ & 0 & 0 & $\begin{array}{l}8 \\
66.7 \%\end{array}$ \\
\hline \multirow[t]{3}{*}{$\begin{array}{l}\text { S. pyogenes } \\
(\mathrm{n}=10)\end{array}$} & $\mathrm{S}$ & $\begin{array}{l}10 \\
100 \%\end{array}$ & $\begin{array}{l}9 \\
90 \%\end{array}$ & $\begin{array}{l}10 \\
100 \%\end{array}$ & $\begin{array}{l}10 \\
100 \%\end{array}$ & $\begin{array}{l}7 \\
70 \%\end{array}$ & $\begin{array}{l}10 \\
100 \%\end{array}$ & $\begin{array}{l}10 \\
100 \%\end{array}$ & $\begin{array}{l}10 \\
100 \%\end{array}$ & $\begin{array}{l}8 \\
80 \%\end{array}$ \\
\hline & I & 0 & 0 & 0 & 0 & 0 & 0 & 0 & 0 & $\begin{array}{l}2 \\
20 \%\end{array}$ \\
\hline & $\mathrm{R}$ & 0 & $\begin{array}{l}1 \\
10 \%\end{array}$ & 0 & 0 & $\begin{array}{l}3 \\
30 \%\end{array}$ & 0 & 0 & 0 & 0 \\
\hline
\end{tabular}

Keys: S = Susceptible or Sensitive; I = Intermediately Susceptible/Sensitive and R = Resistant

Table 6: CLSI interpretive performance standard for antimicrobial disk susceptibility testing [18]

\begin{tabular}{|c|c|c|c|c|}
\hline Antibiotic & Conc. $(\mu \mathrm{g})$ & S & I & $\mathrm{R}$ \\
\hline Amikacin & 30 & $\geq 17$ & $15-16$ & $\leq 14$ \\
\hline Amoxicillin & 30 & $\geq 17$ & - & $\leq 16$ \\
\hline Ampicillin & 10 & $\geq 17$ & - & $\leq 16$ \\
\hline Ceftazidime & 30 & $\geq 21$ & $18-20$ & $\leq 17$ \\
\hline Cefazolin & 30 & $\geq 15$ & - & $\leq 14$ \\
\hline Ceftriaxone & 30 & $\geq 23$ & $20-22$ & $\leq 19$ \\
\hline Chloramphenicol & 30 & $\geq 18$ & $13-17$ & $\leq 12$ \\
\hline Ciprofloxacin & 5 & $\geq 21$ & $16-20$ & $\leq 15$ \\
\hline Clindamycin & 2 & $\geq 21$ & $15-20$ & $\leq 14$ \\
\hline Gentamicin & 10 & $\geq 15$ & $13-14$ & $\leq 12$ \\
\hline Imipenem & 10 & $\geq 23$ & $20-22$ & $\leq 19$ \\
\hline Linezolid & 30 & $\geq 21$ & - & $\leq 20$ \\
\hline Methicillin & 5 & $\geq 19$ & $16-18$ & $\leq 15$ \\
\hline Netilmicin & 30 & $\geq 15$ & $13-14$ & $\leq 12$ \\
\hline Ofloxacin & 5 & $\geq 16$ & $13-15$ & $\leq 12$ \\
\hline Oxacillin & 2 & $\geq 22$ & - & $\leq 21$ \\
\hline Penicillin & 10 & $\geq 15$ & - & $\leq 14$ \\
\hline Piperacilin & 100 & $\geq 21$ & $18-20$ & $\leq 17$ \\
\hline Sulfamethoxazole & 25 & $\geq 30$ & $26-29$ & $\leq 25$ \\
\hline Trimethoprim & 5 & $\geq 16$ & $11-15$ & $\leq 10$ \\
\hline Vancomycin & 30 & $\geq 17$ & $15-16$ & $\leq 14$ \\
\hline
\end{tabular}


Figure 4 showed the percentage susceptibility and resistance of Gram-negative bacteria isolated from diabetic foot ulcers to antibiotics. A high percentage $(76.99 \%)$ of Gram-negative bacteria isolated was susceptible to the conventional antibiotics under study while $22.85 \%$ was resistant and a low percentage $(0.15 \%)$ showed intermediate susceptibility. The percentage susceptibility and resistance of Gram-positive bacteria isolated from diabetic foot ulcers to antibiotics was shown in Figure 5. Gram-positive bacteria showed $93.75 \%$ susceptibility, $1.21 \%$ intermediate susceptibility and $5.01 \%$ resistance to the conventional antibiotics investigated in this study

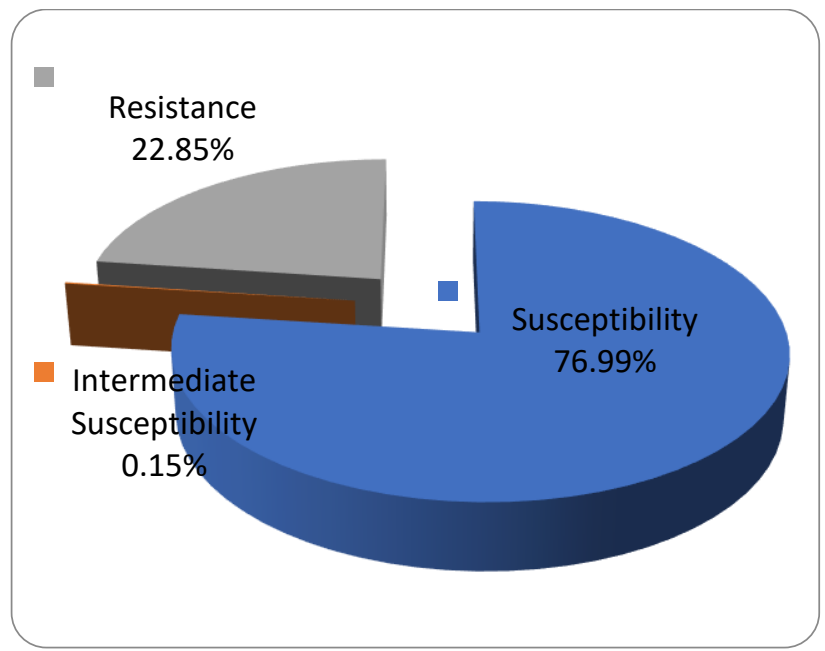

Figure 4: Percentage susceptibility and resistance of Gram-negative bacteria isolated from diabetic foot ulcers to antibiotics

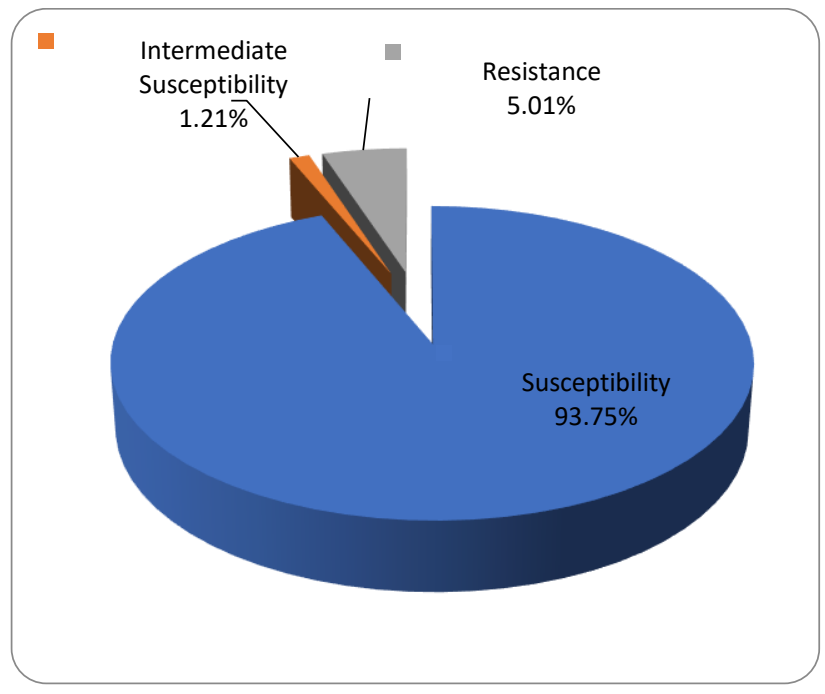

Figure 5: Percentage susceptibility and resistance of Gram-positive bacteria isolated from diabetic foot ulcers to antibiotics
Staphylococcus aureus had the highest percentage occurrence, followed by $P$. aeruginosa among inpatients and out-patients. Among the in-patients, Acinetobacter baumannii had the lowest occurrence while $M$. morganii had the lowest among the outpatients. S. aureus remained the most predominant while $A$. baumannii had the least. This agrees with the results of Perim et al. [19], Ayesha [20] and Pradeep et al. [21]. This result deviated from the reports of Banashankari et al. [22] and Daniel et al. [23] who reported E. coli, next to staphylococci, as the predominant bacteria in foot ulcer. The authors reported some of the bacteria encountered in this study but in different frequency. There were a greater number of Gram-negative bacteria than Grampositive bacteria associated with diabetic foot ulcers and more than one potential pathogen was recovered from the samples analyzed in this study which is in agreement with some other studies [12], [24], [25].

The antibiotic resistance profiles of $S$. aureus strains in this study were similar to the observations made by Joseph et al. [25] and Oates et al. [26] in their study on diabetic foot infection. It, however, differs from the report of Daniel et al. [23] who reported 100\% resistance of $S$. aureus isolates to vancomycin. Vancomycin resistance in Staphylococcus aureus among diabetic patients with foot lesion was also reported by Oates et al. [26]. However, different susceptibility patterns to antibiotics have been shown by other studies and, mostly, vancomycin, amikacin and linezolid have shown good activity against the strains [27] - [30], which is in agreement with findings of the present study.

The challenge of antibiotic resistance is a major public health concern, due to its global dimension and alarming magnitude, although the epidemiology of resistance can exhibit remarkable geographical variability and rapid temporal evolution. The understanding of bacteria associated with diabetic foot infections (DFI) and their antibiotic susceptibility profiles is essential for appropriate treatment and infection eradication. The empirical initiation of antibiotic therapy in patients with serious infections is necessary to prevent systemic invasion by infecting bacteria while awaiting microbiology laboratory results [31].

Clinicians should consider the results of bacterial culture and susceptibility testing in the light of the 
clinical outcome of the infection for the empirical therapy regimen. Knowledge of the characteristics of infection, i.e., the type of bacteria commonly found and the clinical evidence of infections, can help in choosing an appropriate antibiotic, even if the culture reports are not available at the time of initiation of antibiotic therapy [32] - [33].

The $22.85 \%$ resistance exhibited by the bacteria associated with diabetic foot infection in this study is of medical relevance due to the possibility of transmitting those resistant genes to other bacteria sharing the same ecological niche, and thus, impairing the implementation of successful antibiotic therapy [34]. Antimicrobial resistance could be transmitted to the human population, hospitalized patients, and the hospital environment through other sources including animals, plant-based foods, fish, poultry, and other industries in which antibiotics are used for different purposes and may lead to emerging resistant strains of bacteria [35] - [37].

The high antibiotic sensitivity profiles recorded in this study differ from many other studies [38] -[44], where bacterial strains encountered exhibited high resistance to most of the antibiotics investigated. This apparently revealed the variations in antibiotic sensitivity pattern of bacteria isolates based on geographical area. Bacterial strains, encountered in one geograpghical area or country are, in most cases, genetically different from others.

\section{Conclusions}

This study revealed that there is no definite aetiologic bacterial agent for diabetic foot infections and many of the associated bacteria are sensitive to certain antibiotics. Many bacteria that had been previously reported by many authors to be resistant to certain antibiotics were found to be susceptible to the antibiotics used in this study. Thus, many multi-drug resistant bacteria which could complicate the management of diabetic foot infections could be treated by the reported antibiotics. The study showed that many potential pathogens are associated with foot ulcers and which could pose serious health havoc if prompt therapeutic intervention is not made. This finding could assist clinicians to develop antibiotic therapy policy for the early treatment of diabetic foot infections in southwestern Nigeria.

\section{Declarations}

\subsection{Acknowledgements}

Appreciations to the Research Ethics Committees of UCH, Ibadan and OOUTH, Sagamu, Nigeria for the permissions to obtain samples from patients visiting the teaching hospitals.

\subsection{Study Limitations}

There is paucity of information on factors responsible for prevalence of diabetic foot infection in this study. Further research is needed to investigate the patients' attitude with respect to knowledge and practice of chronic wound care. In-depth information gained from current reports will be useful in developing riskassessment model for a larger prospective cohort study. Metagenomic analysis on whole genome sequences of connected tissues is necessary to determine the microbial diversity associated with diabetic foot infections.

\subsection{Ethical Approval}

Ethical approval was given by Research Ethics Committees of UCH, Ibadan and OOUTH, Sagamu to promote this research.

\subsection{Informed Consent}

Informed consent to carry out research and disseminate findings were obtained from participants.

\subsection{Funding Source}

None

\subsection{Competing Interests}

Author declared, no potential conflict of interest exists.

\section{How to Cite this Article}

Bello et al., "Antibiotic Susceptibility Profiles of Bacteria from Diabetic Foot Infections in Selected Teaching Hospitals in Southwestern Nigeria", International Annals of Science, vol. 4, no. 1, pp. 1-13, Jan. 2018. doi: 10.21467/ias.4.1.1-13

\section{References}

[1] J. Jneida, J.P. Lavigneb, B. La Scolaa and N. Cassira, 'The diabetic foot microbiota: A review," Human Microbiome Journal, vol. 5-6, pp. 1-6, Dec. 2017.

[2] World Health Organization [WHO], Global Report on Diabetes. Geneva: April 2016.

[3] N. R. Barshes, M. Sigireddi, J. S. Wrobel, A. Mahankhali, J. L. Robbins, P. Kougias and D. G. Armstrong, "The system of care for the diabetic foot: objectives, outcomes and 
opportunities," Diabetic Foot and Ankle vol. 4, p. 21847. Oct. 2013.

[4] R. E. Mirza, M. M. Fang, E. M. Weinheimer-Haus, W. J. Ennis and T. J. Koh, "Sustained inflammasome activity in macrophages impairs wound healing in type 2 diabetic humans and mice," Diabetes, vol. 63, no. 3, pp. 1103-1114, March 2014.

[5] E. C. Leal, E. Carvalho, A. Tellechea, A. Kafanas, F. Tecilazich, C. Kearney, S. Kuchibhotla, M. E. Auster, E. Kokkotou, D. J. Mooney, F. W. LoGerfo, L. Pradhan-Nabzdyk and A. Veves, "Substance $\mathrm{P}$ promotes wound healing in diabetes by modulating inflammation and macrophage phenotype," The American Journal of Pathology, vol. 185, no. 6, pp. 1638-1648, June 2015.

[6] I. Hsu, L. G. Parkinson, Y. Shen, A. Toro, T. Brown, H. Zhao, R.C. Bleackley and D. J. Granvile, "Serpina3n accelerates tissue repair in a diabetic mouse model of delayed wound healing," Cell Death and Disease, vol. 5, e1458, Oct 2014.

[7] A. Spichler, B. L. Hurwitz, D. G. Armstrong and B. A. Lipsky, "Microbiology of diabetic foot infections: from Louis Pasteur to "crime scene investigation," BMC Medicine. vol. 13, p. 2, Jan. 2015.

[8] J. Moura, J. Rodrigues, M. Goncalves, C. Amaral, M. Lima and E. Carvalho, "Impaired T-cell differentiation in diabetic foot ulceration," Cellular and Molecular Immunology, vol. 14, pp. 758-769, March 2016.

[9] R. D. Wolcott, J. D. Hanson, E. J. Rees, L. D. Koenig, C. D. Phillips, R. A. Wolcott, S. B. Cox and J.S. White, "Analysis of the chronic wound microbiota of 2,963 patients by $16 \mathrm{~S}$ rDNA pyrosequencing," Wound Repair Regen, vol. 24, no. 1, pp. 163-174, Feb 2016.

[10] K. Rahim, S. Saleha, X. Zhu, L. Huo, A. Basit, and O. L. Franco, "Bacterial Contribution in Chronicity of Wounds," Microbial Ecology, vol. 73, no. 3, pp. 710-721, April 2017.

[11] R.E. Pecoraro, G.E. Reiber, E.M. Burgess. "Pathways to diabetic limb amputation: basis for prevention," Diabetes Care, vol. 13, no. 5, pp. 513-521, May 1990.

[12] B.A. Lipsky, A.R. Berendt, P.B. Cornia, J.C. Pile, E.J.G. Peters, D.G. Armstrong, H.G., Deery, J.M. Embil, W.S. Joseph, A.W. Karchmer, M.S. Pinzur and E. Senneville. "2012 infectious diseases society of America clinical practice guideline for the diagnosis and treatment of diabetic foot infections," Clinical Infectious Diseases, vol. 54, no. 12, e132 - e173, June. 2012.

[13] A. Icks, M. Scheer, E. Morbach, J. Genz, B. Haastert, G. Giani, G. Glaeske and F. Hoffman, "Time-dependent impact of diabetes on mortality in patients after major lower extremity amputation." Diabetes Care, vol. 34, pp. 1350-1354, June 2011 .

[14] M. Kerr, "Foot Care for People with Diabetes: The Economic Case for Change," NHS Diabetes, 2012-03. Available at: http://www.diabetes.org.uk/Documents/nhs-

diabetes/footcare/footcare-for-people-with-diabetes.pdf [accessed July 19,2017].

[15] A. Banu, M. M. NoorulHassan, J. Rajkumar, and S. Srinivasa, "Spectrum of bacteria associated with diabetic foot ulcer and biofilm formation: a prospective study," The Australasian Medical Journal, vol. 8, no. 9, pp. 280-285, Sept 2015.

[16] R. Santos, A.S. Veiga, L. Tavares, M. Castanho and M. Oliveira, "Bacterial biofilms in diabetic foot ulcers: potential alternative therapeutics", in Microbial Biofilms - Importance and Applications, ed. D. Dhanasekaran and N. Thajuddin (Rijeka: InTech Press), July 2016. pp. 251-269.

[17] M. Cheesbrough. District laboratory practice in tropical countries. part 2, 2nd ed. UK: Cambridge University, 2006, p. 35-197.
[18] CLSI (Clinical Laboratory Standards Institute), "Performance standards for antimicrobial susceptibility testing" NCCLS approved standard M100-S14, Wayne, PA. USA, II (2), 2016, pp. 298-102.

[19] M. C. Perim, J. C. Borges, S. R. C. Celeste, E. F. Orsolin, R. R. Mendes, G. O. Mendes, R. L. Ferreira, S.C. Carreiro, and M.C. Pranchevicius, "Aerobic bacterial profile and antibiotic resistance in patients with diabetic foot infections," Revista da Sociedade Brasileira de Medicina Tropical, vol. 48, no. 5 , Sept/Oct 2015.

[20] A. Nageen, "The Most Prevalent Organism in Diabetic Foot Ulcers and Its Drug Sensitivity and Resistance to Different Standard Antibiotics," Journal of the College of Physicians and Surgeons Pakistan, vol. 26, no. 4, pp. 293-296, Dec 2016.

[21] M. S. S. Pradeep, K. Vishnuvardhanarao and C. R. Setty, "Bacteriological profile and their Antibiotic Sensitivity Pattern in Diabetic Foot Infections in a Tertiary Care Hospital," Scholars Journal of Applied Medical Sciences, vol. 5, no. 5C pp. 1883-1887, May 2017.

[22] G.S. Banashankari, H.K. Rudresh and A.H. Harsha "Prevalence of Gram Negative Bacteria in Diabetic Foot-A Clinico-Microbiological Study," Al-Ameen Journal of Medical Sciences, vol. 5, no. 3, pp. 224 -232, Sept. 2012.

[23] S.J.C. Daniel, E. Gowthami and S. Sowmiya, "Isolation and identification of bacterial pathogens from wounds of diabetic patients," International Journal of Current Microbiology and Applied Sciences, vol. 2, no. 11, pp. 72-77, 2013.

[24] Z. Mohammad, M. Abida and A. Jamal. "Clinico-bacteriology and risk factors for the diabetic foot infection with multidrug resistant microorganisms in north India." Biology and Medicine, vol. 2, no. 4, pp. 22-34, 2010.

[25] K. Joseph, A. T. George, M. B. Divya and K. K. Ameena, "Diabetic foot infections: characterization and antibiotic resistance pattern of aerobic bacterial isolates in a tertiary care hospital of North Kerala." International Journal of Current Microbiology and Applied Sciences, vol. 6, no. 9, pp. 34933499, Sept 2017.

[26] A. Oates, L. Frank, J.M.B. Andrew, M. Boulton -Citron, J. Ellie, C. Goldstein, C. Vreni- Merriam, A.B. Lipsky and A. Murray. "Bacteriology of moderate-to-severe diabetic foot infections and In vitro activity of antimicrobial agents," Journal of Clinical Microbiology, vol. 45, no. 9, pp. 2819 2828, July 2007.

[27] M. Anvarinejad, G. Pouladfar, A. Japoni, S. Bolandparvaz, Z. Satiary and J. Mardaneh, "Diabetic Foot Infections: antibiotic susceptibility patterns and determination of antibiotic crossresistance in clinical isolates of Enterococcus species during 2012 - 2014 in Shiraz, Iran," Arch Pediatr Infect Dis., vol. 5, no. 2, e37680, April 2017

[28] E. J. Peters, B. A. Lipsky, J. Aragon-Sanchez, E. J. Boyko, M. Diggle, J. M. Embil, S. Kono, L. A. Lavery, E. Senneville, V. Urbancic-Rovan, S. A. Van Asten and W. J. Jeffocate, "Interventions in the management of infection in the foot in diabetes: a systematic review." Diabetes Metabolism Research and Review, vol. 32, no. 1, pp. 145-53, Jan 2016.

[29] M. T. Akhi, R. Ghotaslou, M. Asgharzadeh, M. Varshochi, T. Pirzadeh, M. Y. Memar, A. Z. Bialvaei, H. S. Y. Sofla and N. Alizadeh "Bacterial etiology and antibiotic susceptibility pattern of diabetic foot infections in Tabriz, Iran," GMS Hygiene and Infection Control, vol. 10, no. 2, Feb 2015.

[30] T. G. D. Pemayun, and R. M. Naibaho, "Clinical profile and outcome of diabetic foot ulcer, a view from tertiary care hospital in Semarang, Indonesia." Diabetic Foot and Ankle, vol. 8, no. 1, p. 1312974, May 2017. 
Bello et al., Int. Ann. Sci.; Vol. 4 Issue 1, pp: 1-13, 2018

[31] S. V. Patil and R. R. Mane. Bacterial and clinical profile of diabetic foot ulcer using optimal culture techniques. International Journal of Research in Medical Sciences, vol. 5, no. 2, pp. 496-502, Feb 2017.

[32] A. Karakus, M. Ozkan, M. Karcioglu, R. Ozden, I. Ustun, K. Caliskan, C. Gokce and M. Sahan, "Diabetic foot due to anaphylactic shock: a case report." Archives of Trauma Research, vol. 3, no. 2, e17610, June 2014.

[33] B. Poorabbas, J. Mardaneh, Z. Rezaei, M. Kalani, G. Pouladfar, M. H. Alami, J. Soltani, A. Shamsi-Zadeh, S. Abdoli-Oskooi, M. J. Saffar and A. Alborzi, "Nosocomial Infections: Multicenter surveillance of antimicrobial resistance profile of Staphylococcus aureus and Gram negative rods isolated from blood and other sterile body fluids in Iran." Iranian Journal of Microbiology, vol. 7, no. 3, pp. 127-35, June 2015.

[34] T. Semedo-Lemsaddek, C. Mottola, C. Alves-Barroco, P. Cavaco-Silva, L. Tavares, M. Oliveira, "Characterization of multidrug-resistant diabetic foot ulcer enterococci." Enfermedades Infecciosas y Microbiologia Clinica, vol. 34, no. 2, pp. 114-116, Feb 2015.

[35] J. Mardaneh, and M. M. Soltan-Dallal, "Isolation and identification of $E$. cowanii from powdered infant formula in NICU and determination of antimicrobial susceptibility of isolates. Iranian Journal of Pediatrics, vol. 24, no. 3, pp. 261266, June 2014.

[36] J. Soltani, B. Poorabbas, N. Miri, and J. Mardaneh, "Health care associated infections, antibiotic resistance and clinical outcome: A surveillance study from Sanandaj, Iran.” World Journal of Clinical Cases, vol. 4, no. 3, pp. 63-70, March 2016.

[37] O.O. Bello, H.O. Egberongbe, T.O. Adesetan and A.M. Adenekan, "Antibiotic sensitivity profiles of bacteria isolated from decayed teeth," Scholars Academic Journal of Pharmacy, vol. 2, no. 6, pp. 424-428, Nov 2013.

[38] S. Chang, D.M. Sievert, J.C. Hageman, M.L. Boulton, F.C. Tenover, F.P. Downes, S. Shah, J.T. Rudrik, G.R. Pupp, W.J. Brown, D. Cardo and S.K. Fridkin. "Infection with vancomycin-resistant Staphylococcus aureus containing the vanA resistance gene." New England Journal of Medicine, vol. 348, no. 14, pp. 1342 1347, Apr. 2003.

[39] A. F. de Oliveira and H. D. Oliveira Filho, "Microbiological species and antimicrobial resistance profile in patients with diabetic foot infections," Jornal Vascular Brasileiro, vol. 13, no. 4, pp. 289-293, Dec 2014.

[40] M.F.P. Raymundo, and M.T. Mendoza, "The microbiological features and clinical outcome of diabetic foot infections among patients admitted at UP-PGH." Phillipines Journal of Microbiology and Infectious Diseases, vol. 31, pp. 51-63, 2002.

[41] N. Singh, D.G. Armstrong and B.A. Lipsky, "Preventing foot ulcers in patients with diabetes." The Journal of the American Medical Association, vol. 293, no. 2, pp. 217-28, Jan. 2005.

[42] W.J. Jeffcoate, B.A. Lipsky, A.R. Berendt, P.R. Cavanagh, S.A. Bus, E.J. Peters, W.H. van Houtum, G.D. Valk and K. Bakker, "Unresolved issues in the management of ulcers of the foot in diabetes." Diabetic Medicine, vol. 25, vol. 12, pp.13801389, Jan 2009.

[43] B. M. Pabithadevi, V. K. Rajendran and M. Sadana, "Clinical Outcomes of Diabetic Foot and Its Management," IOSR Journal of Dental and Medical Sciences, vol. 15, no. 1, pp. 1015, Jan 2016.

[44] X. Xie, Y. Bao, L. Ni, D. Liu, S. Niu, H. Lin, H. Li, C. Duan, L. Yan, S. Huang and Z. Luo, "Bacterial profile and antibiotic resistance in patients with diabetic foot ulcer in Guangzhou, Southern China: focus on the differences among different
Wagner's Grades, IDSA/IWGDF grades, and ulcer types. International Journal of Endocrinology, vol. 2017, Article ID 8694903, pp. 1 - 12, July 2017.

Publish your research article in AIJR journals-

$\checkmark$ Online Submission and Tracking

$\checkmark$ Peer-Reviewed

$\checkmark$ Rapid decision

$\checkmark$ Immediate Publication after acceptance

$\checkmark \quad$ Articles freely available online

$\checkmark \quad$ Retain full copyright of your article.

Submit your article at journals.aijr.in

Publish your books with AIJR publisher-

$\checkmark$ Publish with ISBN and DOI.

$\checkmark$ Publish Thesis/Dissertation as a Book.

$\checkmark$ Publish Monograph.

$\checkmark$ Publish Edited Volume/ Book.

$\checkmark$ Publish Conference Proceedings

$\checkmark \quad$ Retain full copyright of your books.

Submit your manuscript at books.aijr.org 